\title{
ON A MAX TYPE RECURSIVE SEQUENCE OF ORDER THREE
}

\author{
E. M. ELSAYED
}

Received 21 November, 2013

\begin{abstract}
Our aim in this paper is to investigate the behavior of the solution of the following max type difference equation of order three

$$
x_{n+1}=\max \left\{\frac{A_{n}}{x_{n}}, x_{n-2}\right\}, n=0,1, \ldots,
$$

where the initial conditions $x_{-2}, x_{-1}, x_{0}$ are arbitrary positive real numbers and $\left\{A_{n}\right\}_{n=0}^{\infty}$ is a
\end{abstract} periodic sequence of period two.

2010 Mathematics Subject Classification: 39A10

Keywords: solution of difference equations, recursive sequences, max-type difference equation, periodic solution, boundedness

\section{INTRODUCTION}

Our purpose in this paper is to study the behavior of the solution of the following max type difference equation

$$
x_{n+1}=\max \left\{\frac{A_{n}}{x_{n}}, x_{n-2}\right\}, \quad n=0,1, \ldots,
$$

where the initial conditions $x_{-2}, x_{-1}, x_{0}$ are arbitrary positive real numbers and $\left\{A_{n}\right\}_{n=0}^{\infty}$ is a periodic sequence of period two.

Nonlinear rational difference equations are of great importance in their own right because diverse nonlinear phenomena occurring in science and engineering can be modeled by such equations. Furthermore, the results about such equations offer prototypes towards the development of the basic theory of nonlinear difference equations. See $[8-10,23,24,26]$.

The study of max-type difference equations attracted a considerable attention recently, see, for example, $[1,5,7,13-16]$ and the references therein. This type of difference equations stem from, for example, certain models in automatic control theory (see [17] and [18]). In the beginning of the study of these equations the experts have been focused on the investigation of the behavior of some particular cases 
of the following difference equation

$$
x_{n}=\max \left\{\frac{A_{n}^{(1)}}{x_{n-1}}, \frac{A_{n}^{(2)}}{x_{n-2}}, \ldots, \frac{A_{n}^{(k)}}{x_{n-k}}\right\}, \quad n \in \mathbb{N}_{0},
$$

where $k \in \mathbb{N}, A_{n}^{(i)}, i=1, \ldots, k$, are real sequences (mostly constant or periodic) and the initial values $x_{-1}, \ldots, x_{-k}$ are different from zero (see, e.g., $[2,3,6,15]$ and the references therein).

Cinar et al. [4] dealt with the positive solutions of the difference equation

$$
x_{n+1}=\max \left\{\frac{A}{x_{n}^{2}}, \frac{B x_{n-1}}{x_{n} x_{n-2}^{2}}\right\} .
$$

Recently, in the paper [12] it was showed that every solution of the third-order maxtype difference equation (1.1), where the initial conditions are arbitrary nonzero real numbers and $A_{n}=$ constant $\in R$, is eventually periodic with period three.

Also, in [11] we proved that every positive solution to the same third order nonautonomous max-type difference equation (1.1), with $\left\{A_{n}\right\}$ is a three-periodic sequence of positive numbers, is periodic with period three. The same result was proved for the same equation but with min-type difference equation.

Simsek et al. [19] investigated the solutions of the following difference equation

$$
x_{n+1}=\max \left\{x_{n-1}, \frac{1}{x_{n-1}}\right\} .
$$

Also, Simsek [20] studied the behavior of the solutions of the following system of difference equations

$$
x_{n+1}=\max \left\{\frac{A}{x_{n}}, \frac{y_{n}}{x_{n}}\right\}, y_{n+1}=\max \left\{\frac{A}{y_{n}}, \frac{x_{n}}{y_{n}}\right\} .
$$

Stevic [21] studied the boundedness and global attractivity for the positive solutions of the difference equation

$$
x_{n+1}=\max \left\{c, \frac{x_{n}^{p}}{x_{n-1}^{p}}\right\} .
$$

In [25] Yalcinkaya et al. investigated the periodic nature of the solution of the maxtype difference equation

$$
x_{n+1}=\max \left\{x_{n}, A\right\} / x_{n}^{2} x_{n-1} .
$$

See also [22], [27].

Definition 1. A sequence $\left\{x_{n}\right\}_{n=-k}^{\infty}$ is said to be eventually periodic with period $p$ if there is $n_{0} \in\{-k, \ldots,-1,0,1, \ldots\}$ such that $x_{n+p}=x_{n}$ for all $n \geq n_{0}$. If $n_{0}=-k$, then we say that the sequence $\left\{x_{n}\right\}_{n=-k}^{\infty}$ is periodic with period $p$. 
Remark 1. Note that if $A_{n}=0$, then Eq. (1.1) becomes $x_{n+1}=x_{n-2}$, from which it follows that every solution is periodic with period three. Hence, in the sequel we will consider the case $A_{n} \neq 0$.

Remark 2. Note that if $A_{n}=A$, then Eq. (1.1) becomes $x_{n+1}=\max \left\{\frac{A}{x_{n}}, x_{n-2}\right\}$, it was shown that this equation is periodic with period three see [12]. Hence, in the sequel we will consider the case $A_{n}=\left\{A_{0}, A_{1}, A_{0}, A_{1}, \ldots\right\}, A_{1} \neq A_{0}$.

\section{MAIN RESULTS}

\subsection{The Case $A_{0}>A_{1}$}

Theorem 1. Consider the difference equation (1.1) with $A_{0}>A_{1}$. Then every solution of Eq. (1.1) is eventually periodic with period three.

Proof. From Eq.(1.1), we see that

$$
x_{1}=\max \left\{\frac{A_{0}}{x_{0}}, x_{-2}\right\} .
$$

We consider two cases

Case $\left(a_{1}\right)$ If $x_{-2} x_{0}<A_{0}$, then $x_{1}=\frac{A_{0}}{x_{0}}$ and

$$
x_{2}=\max \left\{\frac{A_{1}}{x_{1}}, x_{-1}\right\}=\max \left\{\frac{A_{1} x_{0}}{A_{0}}, x_{-1}\right\} .
$$

$\left(a_{11}\right)$ If $x_{-1} A_{0}<A_{1} x_{0}$, then $x_{2}=\frac{A_{1} x_{0}}{A_{0}}$ and

$$
x_{3}=\max \left\{\frac{A_{2}}{x_{2}}, x_{0}\right\}=\max \left\{\frac{A_{0}^{2}}{A_{1} x_{0}}, x_{0}\right\} \text {. }
$$

$$
\begin{aligned}
& \left(a_{111}\right) \text { If } A_{1} x_{0}^{2}<A_{0}^{2} \text {, then } x_{3}=\frac{A_{0}^{2}}{A_{1} x_{0}} \text { and } \\
& x_{4}=\max \left\{\frac{A_{3}}{x_{3}}, x_{1}\right\}=\max \left\{\frac{A_{1}^{2} x_{0}}{A_{0}^{2}}, \frac{A_{0}}{x_{0}}\right\}=\frac{A_{0}}{x_{0}}, \\
& \text { (since } \left.A_{1} x_{0}^{2}<A_{0}^{2} \Rightarrow A_{1}^{2} x_{0}^{2}<A_{0} A_{1} x_{0}^{2}<A_{0}^{3}\right) \\
& x_{5}=\max \left\{\frac{A_{4}}{x_{4}}, x_{2}\right\}=\max \left\{x_{0}, \frac{A_{1} x_{0}}{A_{0}}\right\}=x_{0}, \\
& x_{6}=\max \left\{\frac{A_{5}}{x_{5}}, x_{3}\right\}=\max \left\{\frac{A_{1}}{x_{0}}, \frac{A_{0}^{2}}{A_{1} x_{0}}\right\}=\frac{A_{0}^{2}}{A_{1} x_{0}}, \\
& x_{7}=\max \left\{\frac{A_{6}}{x_{6}}, x_{4}\right\}=\max \left\{\frac{A_{1} x_{0}}{A_{0}}, \frac{A_{0}}{x_{0}}\right\}=\frac{A_{0}}{x_{0}},
\end{aligned}
$$




$$
\begin{aligned}
& x_{8}=\max \left\{\frac{A_{7}}{x_{7}}, x_{5}\right\}=\max \left\{\frac{A_{1} x_{0}}{A_{0}}, x_{0}\right\}=x_{0}, \\
& x_{9}=\max \left\{\frac{A_{8}}{x_{8}}, x_{6}\right\}=\max \left\{\frac{A_{0}}{x_{0}}, \frac{A_{0}^{2}}{A_{1} x_{0}}\right\}=\frac{A_{0}^{2}}{A_{1} x_{0}},
\end{aligned}
$$

and the solution has the following form

$$
\left\{\frac{A_{0}^{2}}{A_{1} x_{0}}, \frac{A_{0}}{x_{0}}, x_{0}, \frac{A_{0}^{2}}{A_{1} x_{0}}, \frac{A_{0}}{x_{0}}, x_{0}, \frac{A_{0}^{2}}{A_{1} x_{0}}, \frac{A_{0}}{x_{0}}, \ldots\right\} .
$$

$\left(a_{112}\right)$ If $A_{1} x_{0}^{2}>A_{0}^{2}$, then $x_{3}=x_{0}$ and

$$
\begin{aligned}
& x_{4}=\max \left\{\frac{A_{3}}{x_{3}}, x_{1}\right\}=\max \left\{\frac{A_{1}}{x_{0}}, \frac{A_{0}}{x_{0}}\right\}=\frac{A_{0}}{x_{0}}, \\
& x_{5}=\max \left\{\frac{A_{4}}{x_{4}}, x_{2}\right\}=\max \left\{x_{0}, \frac{A_{1} x_{0}}{A_{0}}\right\}=x_{0}, \\
& x_{6}=\max \left\{\frac{A_{5}}{x_{5}}, x_{3}\right\}=\max \left\{\frac{A_{1}}{x_{0}}, x_{0}\right\}=x_{0},
\end{aligned}
$$

(since $A_{1} x_{0}^{2}>A_{0}^{2}>A_{1}^{2}$ )

$$
\begin{aligned}
& x_{7}=\max \left\{\frac{A_{6}}{x_{6}}, x_{4}\right\}=\max \left\{\frac{A_{0}}{x_{0}}, \frac{A_{0}}{x_{0}}\right\}=\frac{A_{0}}{x_{0}}, \\
& x_{8}=\max \left\{\frac{A_{7}}{x_{7}}, x_{5}\right\}=\max \left\{\frac{A_{1} x_{0}}{A_{0}}, x_{0}\right\}=x_{0}, \\
& x_{9}=\max \left\{\frac{A_{8}}{x_{8}}, x_{6}\right\}=\max \left\{\frac{A_{0}}{x_{0}}, x_{0}\right\}=x_{0},
\end{aligned}
$$

(since $A_{0} x_{0}^{2}>A_{1} x_{0}^{2}>A_{0}^{2}$ )

then the solution is eventually periodic with period three. Moreover, it has the following form

$$
\left\{\frac{A_{0}}{x_{0}}, x_{0}, x_{0}, \frac{A_{0}}{x_{0}}, x_{0}, x_{0}, \frac{A_{0}}{x_{0}}, \ldots\right\} .
$$

$\left(a_{12}\right)$ If $x_{-1} A_{0}>A_{1} x_{0}$, then $x_{2}=x_{-1}$ and

$$
x_{3}=\max \left\{\frac{A_{2}}{x_{2}}, x_{0}\right\}=\max \left\{\frac{A_{0}}{x_{-1}}, x_{0}\right\} .
$$

$\left(a_{121}\right)$ If $x_{0}<\frac{A_{0}}{x_{-1}}$, then $x_{3}=\frac{A_{0}}{x_{-1}}$ and

$$
x_{4}=\max \left\{\frac{A_{3}}{x_{3}}, x_{1}\right\}=\max \left\{\frac{A_{1} x_{-1}}{A_{0}}, \frac{A_{0}}{x_{0}}\right\}=\frac{A_{0}}{x_{0}},
$$




$$
\begin{aligned}
&\left(\text { since } x_{0}<\frac{A_{0}}{x_{-1}}\right.\left.\Rightarrow A_{1} x_{0}<\frac{A_{1} A_{0}}{x_{-1}}<\frac{A_{0}^{2}}{x_{-1}}\right), \\
& x_{5}=\max \left\{\frac{A_{4}}{x_{4}}, x_{2}\right\}=\max \left\{x_{0}, x_{-1}\right\},
\end{aligned}
$$

$\left(a_{1211}\right)$ If $x_{0}<x_{-1}$, then $x_{5}=x_{-1}$ and

$$
\begin{aligned}
& x_{6}=\max \left\{\frac{A_{5}}{x_{5}}, x_{3}\right\}=\max \left\{\frac{A_{1}}{x_{-1}}, \frac{A_{0}}{x_{-1}}\right\}=\frac{A_{0}}{x_{-1}}, \\
& x_{7}=\max \left\{\frac{A_{6}}{x_{6}}, x_{4}\right\}=\max \left\{x_{-1}, \frac{A_{0}}{x_{0}}\right\}=\frac{A_{0}}{x_{0}}, \\
& x_{8}=\max \left\{\frac{A_{7}}{x_{7}}, x_{5}\right\}=\max \left\{\frac{A_{1} x_{0}}{A_{0}}, x_{-1}\right\}=x_{-1}, \\
& x_{9}=\max \left\{\frac{A_{8}}{x_{8}}, x_{6}\right\}=\max \left\{\frac{A_{1}}{x_{-1}}, \frac{A_{0}}{x_{-1}}\right\}=\frac{A_{0}}{x_{-1}} .
\end{aligned}
$$

Clearly, the derived solution is eventually periodic with period three and takes the form

$$
\left\{\frac{A_{0}}{x_{0}}, x_{-1}, \frac{A_{0}}{x_{-1}}, \frac{A_{0}}{x_{0}}, x_{-1}, \frac{A_{0}}{x_{-1}}, \ldots\right\} .
$$

$\left(a_{1212}\right)$ If $x_{0}>x_{-1}$, then $x_{5}=x_{0}$ and

$$
\begin{gathered}
x_{6}=\max \left\{\frac{A_{5}}{x_{5}}, x_{3}\right\}=\max \left\{\frac{A_{1}}{x_{0}}, \frac{A_{0}}{x_{-1}}\right\}=\frac{A_{0}}{x_{-1}}, \\
\left(\text { since } x_{0}>x_{-1} \Rightarrow A_{0} x_{0}>A_{0} x_{-1}>A_{1} x_{-1}\right), \\
x_{7}=\max \left\{\frac{A_{6}}{x_{6}}, x_{4}\right\}=\max \left\{x_{-1}, \frac{A_{0}}{x_{0}}\right\}=\frac{A_{0}}{x_{0}}, \\
x_{8}=\max \left\{\frac{A_{7}}{x_{7}}, x_{5}\right\}=\max \left\{\frac{A_{1} x_{0}}{A_{0}}, x_{0}\right\}=x_{0}, \\
x_{9}=\max \left\{\frac{A_{8}}{x_{8}}, x_{6}\right\}=\max \left\{\frac{A_{0}}{x_{0}}, \frac{A_{0}}{x_{-1}}\right\}=\frac{A_{0}}{x_{-1}},
\end{gathered}
$$

we obtain that the solution is eventually periodic with period three. It has the following form

$$
\left\{\frac{A_{0}}{x_{-1}}, \frac{A_{0}}{x_{0}}, x_{0}, \frac{A_{0}}{x_{-1}}, \frac{A_{0}}{x_{0}}, x_{0}, \ldots\right\} .
$$

$\left(a_{122}\right)$ If $A_{0}<x_{-1} x_{0}$, then $x_{3}=x_{0}$ and

$$
x_{4}=\max \left\{\frac{A_{3}}{x_{3}}, x_{1}\right\}=\max \left\{\frac{A_{1}}{x_{0}}, \frac{A_{0}}{x_{0}}\right\}=\frac{A_{0}}{x_{0}},
$$




$$
x_{5}=\max \left\{\frac{A_{4}}{x_{4}}, x_{2}\right\}=\max \left\{x_{0}, x_{-1}\right\},
$$

$\left(a_{1221}\right)$ If $x_{0}<x_{-1}$, then $x_{5}=x_{-1}$ and

$$
\begin{gathered}
x_{6}=\max \left\{\frac{A_{5}}{x_{5}}, x_{3}\right\}=\max \left\{\frac{A_{1}}{x_{-1}}, x_{0}\right\}=x_{0}, \\
\left(\text { since } x_{0}>\frac{A_{0}}{x_{-1}}>\frac{A_{1}}{x_{-1}}\right), \\
x_{7}=\max \left\{\frac{A_{6}}{x_{6}}, x_{4}\right\}=\max \left\{\frac{A_{0}}{x_{0}}, \frac{A_{0}}{x_{0}}\right\}=\frac{A_{0}}{x_{0}}, \\
x_{8}=\max \left\{\frac{A_{7}}{x_{7}}, x_{5}\right\}=\max \left\{\frac{A_{1} x_{0}}{A_{0}}, x_{-1}\right\}=x_{-1}, \\
\left(\text { since } x_{0}<x_{-1} \Rightarrow A_{1} x_{0}<A_{0} x_{0}<A_{0} x_{-1}\right), \\
x_{9}=\max \left\{\frac{A_{8}}{x_{8}}, x_{6}\right\}=\max \left\{\frac{A_{0}}{x_{-1}}, x_{0}\right\}=x_{0},
\end{gathered}
$$

the presented solution has the form

$$
\left\{x_{-1}, x_{0}, \frac{A_{0}}{x_{0}}, x_{-1}, x_{0}, \frac{A_{0}}{x_{0}}, \ldots\right\} .
$$

$\left(a_{1222}\right)$ If $x_{0}>x_{-1}$, then $x_{5}=x_{0}$ and

$$
\begin{aligned}
& x_{6}=\max \left\{\frac{A_{5}}{x_{5}}, x_{3}\right\}=\max \left\{\frac{A_{1}}{x_{0}}, x_{0}\right\}=x_{0}, \\
&\left(\text { since } x_{0}>\frac{A_{0}}{x_{-1}}>\frac{A_{1}}{x_{-1}} \& x_{0}>x_{-1} \Rightarrow x_{0}^{2}>x_{0} x_{-1}>A_{1}\right), \\
& x_{7}=\max \left\{\frac{A_{6}}{x_{6}}, x_{4}\right\}=\max \left\{\frac{A_{0}}{x_{0}}, \frac{A_{0}}{x_{0}}\right\}=\frac{A_{0}}{x_{0}}, \\
& x_{8}=\max \left\{\frac{A_{7}}{x_{7}}, x_{5}\right\}=\max \left\{\frac{A_{1} x_{0}}{A_{0}}, x_{0}\right\}=x_{0}, \\
& x_{9}=\max \left\{\frac{A_{8}}{x_{8}}, x_{6}\right\}=\max \left\{\frac{A_{0}}{x_{0}}, x_{0}\right\}=x_{0},
\end{aligned}
$$

we obtain that the solution is eventually periodic with period three and the developed solution has the following form

$$
\left\{x_{0}, \frac{A_{0}}{x_{0}}, x_{0}, x_{0}, \frac{A_{0}}{x_{0}}, x_{0}, x_{0}, \ldots\right\} .
$$

Case $\left(a_{2}\right)$ If $x_{-2} x_{0}>A_{0}$, then $x_{1}=x_{-2}$ and

$$
x_{2}=\max \left\{\frac{A_{1}}{x_{1}}, x_{-1}\right\}=\max \left\{\frac{A_{1}}{x_{-2}}, x_{-1}\right\} .
$$


$\left(a_{21}\right)$ If $x_{-1} x_{-2}<A_{1}$, then $x_{2}=\frac{A_{1}}{x_{-2}}$ and

$$
x_{3}=\max \left\{\frac{A_{2}}{x_{2}}, x_{0}\right\}=\max \left\{\frac{A_{0} x_{-2}}{A_{1}}, x_{0}\right\} .
$$

$\left(a_{211}\right)$ If $A_{1} x_{0}<A_{0} x_{-2}$, then $x_{3}=\frac{A_{0} x_{-2}}{A_{1}}$ and

$$
x_{4}=\max \left\{\frac{A_{3}}{x_{3}}, x_{1}\right\}=\max \left\{\frac{A_{1}^{2}}{A_{0} x_{-2}}, x_{-2}\right\}=x_{-2},
$$

(since $A_{1} x_{0}<A_{0} x_{-2} \Rightarrow \frac{1}{x_{0}}>\frac{A_{1}}{A_{0} x_{-2}} \& x_{-2}>\frac{A_{0}}{x_{0}}$

$$
\begin{aligned}
\left.\Rightarrow x_{-2}>\frac{A_{0}}{x_{0}}>\frac{A_{0} A_{1}}{A_{0} x_{-2}}>\frac{A_{1}^{2}}{A_{0} x_{-2}}\right), \\
x_{5}=\max \left\{\frac{A_{4}}{x_{4}}, x_{2}\right\}=\max \left\{\frac{A_{0}}{x_{-2}}, \frac{A_{1}}{x_{-2}}\right\}=\frac{A_{0}}{x_{-2}}, \\
x_{6}=\max \left\{\frac{A_{5}}{x_{5}}, x_{3}\right\}=\max \left\{\frac{A_{1} x_{-2}}{A_{0}}, \frac{A_{0} x_{-2}}{A_{1}}\right\}=\frac{A_{0} x_{-2}}{A_{1}}, \\
x_{7}=\max \left\{\frac{A_{6}}{x_{6}}, x_{4}\right\}=\max \left\{\frac{A_{1}}{x_{-2}}, x_{-2}\right\}=x_{-2},
\end{aligned}
$$

(since $A_{0} x_{-2}^{2}>A_{0} A_{1}$ ),

$$
\begin{aligned}
& x_{8}=\max \left\{\frac{A_{7}}{x_{7}}, x_{5}\right\}=\max \left\{\frac{A_{1}}{x_{-2}}, \frac{A_{0}}{x_{-2}}\right\}=\frac{A_{0}}{x_{-2}}, \\
& x_{9}=\max \left\{\frac{A_{8}}{x_{8}}, x_{6}\right\}=\max \left\{x_{-2}, \frac{A_{0} x_{-2}}{A_{1}}\right\}=\frac{A_{0} x_{-2}}{A_{1}},
\end{aligned}
$$

the resulting solution is then eventually periodic with period three in the closed form

$$
\left\{\frac{A_{0} x_{-2}}{A_{1}}, x_{-2}, \frac{A_{0}}{x_{-2}}, \frac{A_{0} x_{-2}}{A_{1}}, x_{-2}, \frac{A_{0}}{x_{-2}}, \ldots\right\} .
$$

$\left(a_{212}\right)$ If $A_{1} x_{0}>A_{0} x_{-2}$, then $x_{3}=x_{0}$ and

$$
x_{4}=\max \left\{\frac{A_{3}}{x_{3}}, x_{1}\right\}=\max \left\{\frac{A_{1}}{x_{0}}, x_{-2}\right\}=x_{-2},
$$

(since $x_{0} x_{-2}>A_{0}>A_{1}$ ),

$$
\begin{aligned}
& x_{5}=\max \left\{\frac{A_{4}}{x_{4}}, x_{2}\right\}=\max \left\{\frac{A_{0}}{x_{-2}}, \frac{A_{1}}{x_{-2}}\right\}=\frac{A_{0}}{x_{-2}}, \\
& x_{6}=\max \left\{\frac{A_{5}}{x_{5}}, x_{3}\right\}=\max \left\{\frac{A_{1} x_{-2}}{A_{0}}, x_{0}\right\}=x_{0},
\end{aligned}
$$


(since $A_{0} x_{0}>A_{1} x_{0}>A_{0} x_{-2}>A_{1} x_{-2}$ ),

$$
\begin{aligned}
& x_{7}=\max \left\{\frac{A_{6}}{x_{6}}, x_{4}\right\}=\max \left\{\frac{A_{0}}{x_{0}}, x_{-2}\right\}=x_{-2}, \\
& x_{8}=\max \left\{\frac{A_{7}}{x_{7}}, x_{5}\right\}=\max \left\{\frac{A_{1}}{x_{-2}}, \frac{A_{0}}{x_{-2}}\right\}=\frac{A_{0}}{x_{-2}}, \\
& x_{9}=\max \left\{\frac{A_{8}}{x_{8}}, x_{6}\right\}=\max \left\{x_{-2}, x_{0}\right\}=x_{0},
\end{aligned}
$$

(since $A_{1} x_{0}>A_{0} x_{-2}>A_{1} x_{-2}$ ).

Thus, the solution is

$$
\left\{x_{-2}, \frac{A_{0}}{x_{-2}}, x_{0}, x_{-2}, \frac{A_{0}}{x_{-2}}, x_{0}, x_{-2}, \ldots\right\} .
$$

$\left(a_{22}\right)$ If $x_{-1} x_{-2}>A_{1}$, then $x_{2}=x_{-1}$ and

$$
x_{3}=\max \left\{\frac{A_{2}}{x_{2}}, x_{0}\right\}=\max \left\{\frac{A_{0}}{x_{-1}}, x_{0}\right\} .
$$

$\left(a_{221}\right)$ If $x_{0} x_{-1}<A_{0}$, then $x_{3}=\frac{A_{0}}{x_{-1}}$ and

$$
\begin{aligned}
& x_{4}=\max \left\{\frac{A_{3}}{x_{3}}, x_{1}\right\}=\max \left\{\frac{A_{1} x_{-1}}{A_{0}}, x_{-2}\right\}=x_{-2}, \\
&\left(\text { since } x_{-2}>\frac{A_{0}}{x_{0}}>\frac{x_{0} x_{-1}}{x_{0}} \Rightarrow A_{0} x_{-2}>A_{1} x_{-2}>A_{0} x_{-1}>A_{1} x_{-1}\right), \\
& x_{5}=\max \left\{\frac{A_{4}}{x_{4}}, x_{2}\right\}=\max \left\{\frac{A_{0}}{x_{-2}}, x_{-1}\right\},
\end{aligned}
$$

$\left(a_{2211}\right)$ If $\frac{A_{0}}{x_{-2}}<x_{-1}$, then $x_{5}=x_{-1}$ and

$$
\begin{aligned}
& x_{6}=\max \left\{\frac{A_{5}}{x_{5}}, x_{3}\right\}=\max \left\{\frac{A_{1}}{x_{-1}}, \frac{A_{0}}{x_{-1}}\right\}=\frac{A_{0}}{x_{-1}}, \\
& x_{7}=\max \left\{\frac{A_{6}}{x_{6}}, x_{4}\right\}=\max \left\{x_{-1}, x_{-2}\right\}=x_{-2}, \\
& x_{8}=\max \left\{\frac{A_{7}}{x_{7}}, x_{5}\right\}=\max \left\{\frac{A_{1}}{x_{-2}}, x_{-1}\right\}=x_{-1}, \\
& x_{9}=\max \left\{\frac{A_{8}}{x_{8}}, x_{6}\right\}=\max \left\{\frac{A_{0}}{x_{-1}}, \frac{A_{0}}{x_{-1}}\right\}=\frac{A_{0}}{x_{-1}},
\end{aligned}
$$


this provides an eventually periodic with period three, which takes the form

$$
\left\{x_{-2}, x_{-1}, \frac{A_{0}}{x_{-1}}, x_{-2}, x_{-1}, \frac{A_{0}}{x_{-1}}, \ldots\right\} \text {. }
$$

$\left(a_{2212}\right)$ If $\frac{A_{0}}{x_{-2}}>x_{-1}$, then $x_{5}=\frac{A_{0}}{x_{-2}}$ and

$$
\begin{gathered}
x_{6}=\max \left\{\frac{A_{5}}{x_{5}}, x_{3}\right\}=\max \left\{\frac{A_{1} x_{-2}}{A_{0}}, \frac{A_{0}}{x_{-1}}\right\}=\frac{A_{0}}{x_{-1}}, \\
\left(\text { since } x_{-2} x_{-1}<A_{0} \Rightarrow A_{1} x_{-2} x_{-1}<A_{1} A_{0}<A_{0}^{2}\right), \\
x_{7}=\max \left\{\frac{A_{6}}{x_{6}}, x_{4}\right\}=\max \left\{x_{-1}, x_{-2}\right\}=x_{-2}, \\
x_{8}=\max \left\{\frac{A_{7}}{x_{7}}, x_{5}\right\}=\max \left\{\frac{A_{1}}{x_{-2}}, \frac{A_{0}}{x_{-2}}\right\}=\frac{A_{0}}{x_{-2}}, \\
x_{9}=\max \left\{\frac{A_{8}}{x_{8}}, x_{6}\right\}=\max \left\{\frac{A_{1} x_{-2}}{A_{0}}, \frac{A_{0}}{x_{-1}}\right\}=\frac{A_{0}}{x_{-1}},
\end{gathered}
$$

obviously, the solution is eventually periodic with period three, which can be expressed in the form

$$
\left\{\frac{A_{0}}{x_{-1}}, x_{-2}, \frac{A_{0}}{x_{-2}}, \frac{A_{0}}{x_{-1}}, x_{-2}, \frac{A_{0}}{x_{-2}}, \ldots\right\} .
$$

$\left(a_{222}\right)$ If $A_{0}<x_{-1} x_{0}$, then $x_{3}=x_{0}$ and

$$
\begin{gathered}
x_{4}=\max \left\{\frac{A_{3}}{x_{3}}, x_{1}\right\}=\max \left\{\frac{A_{1}}{x_{0}}, x_{-2}\right\}=x_{-2}, \\
\left(\text { since } x_{-2} x_{0}>A_{0}>A_{1}\right) \\
x_{5}=\max \left\{\frac{A_{4}}{x_{4}}, x_{2}\right\}=\max \left\{\frac{A_{0}}{x_{-2}}, x_{-1}\right\},
\end{gathered}
$$

$\left(a_{2221}\right)$ If $\frac{A_{0}}{x_{-2}}<x_{-1}$, then $x_{5}=x_{-1}$ and

$$
\begin{aligned}
& x_{6}=\max \left\{\frac{A_{5}}{x_{5}}, x_{3}\right\}=\max \left\{\frac{A_{1}}{x_{-1}}, x_{0}\right\}=x_{0}, \\
& x_{7}=\max \left\{\frac{A_{6}}{x_{6}}, x_{4}\right\}=\max \left\{\frac{A_{0}}{x_{0}}, x_{-2}\right\}=x_{-2}, \\
& x_{8}=\max \left\{\frac{A_{7}}{x_{7}}, x_{5}\right\}=\max \left\{\frac{A_{1}}{x_{-2}}, x_{-1}\right\}=x_{-1}, \\
& x_{9}=\max \left\{\frac{A_{8}}{x_{8}}, x_{6}\right\}=\max \left\{\frac{A_{0}}{x_{-1}}, x_{0}\right\}=x_{0},
\end{aligned}
$$


this in turn gives the eventually periodic solution with period three

$$
\left\{x_{-2}, x_{-1}, x_{0}, x_{-2}, x_{-1}, x_{0}, \ldots\right\} .
$$

$\left(a_{2222}\right)$ If $\frac{A_{0}}{x_{-2}}>x_{-1}$, then $x_{5}=\frac{A_{0}}{x_{-2}}$ and

$$
\begin{gathered}
x_{6}=\max \left\{\frac{A_{5}}{x_{5}}, x_{3}\right\}=\max \left\{\frac{A_{1} x_{-2}}{A_{0}}, x_{0}\right\}=x_{0}, \\
\left(\text { since } x_{0}>\frac{A_{0}}{x_{-1}}>x_{-2} \Rightarrow A_{0} x_{0}>A_{0} x_{-2}>A_{1} x_{-2}\right), \\
x_{7}=\max \left\{\frac{A_{6}}{x_{6}}, x_{4}\right\}=\max \left\{\frac{A_{0}}{x_{0}}, x_{-2}\right\}=x_{-2}, \\
x_{8}=\max \left\{\frac{A_{7}}{x_{7}}, x_{5}\right\}=\max \left\{\frac{A_{1}}{x_{-2}}, \frac{A_{0}}{x_{-2}}\right\}=\frac{A_{0}}{x_{-2}}, \\
x_{9}=\max \left\{\frac{A_{8}}{x_{8}}, x_{6}\right\}=\max \left\{x_{-2}, x_{0}\right\}=x_{0},
\end{gathered}
$$

this gives the solution

$$
\left\{x_{0}, x_{-2}, \frac{A_{0}}{x_{-2}}, x_{0}, x_{-2}, \frac{A_{0}}{x_{-2}}, \ldots\right\} .
$$

2.2. The Case $A_{1}>A_{0}$

Theorem 2. Consider the difference equation (1.1) for $A_{1}>A_{0}$. Then the solution is periodic with period three or the equation has unboundedness solution takes the form:

$$
\begin{aligned}
\left\{x_{2 n-1}\right\}_{n=1}^{\infty} & =\left\{x_{-2}, x_{-2}\left(\frac{A_{0}}{A_{1}}\right), x_{-2}\left(\frac{A_{0}}{A_{1}}\right)^{2}, x_{-2}\left(\frac{A_{0}}{A_{1}}\right)^{3}, \ldots\right\}, \\
\left\{x_{2 n}\right\}_{n=1}^{\infty} & =\left\{\frac{A_{1}}{x_{-2}}, \frac{A_{1}}{x_{-2}}\left(\frac{A_{1}}{A_{0}}\right), \frac{A_{1}}{x_{-2}}\left(\frac{A_{1}}{A_{0}}\right)^{2}, \frac{A_{1}}{x_{-2}}\left(\frac{A_{1}}{A_{0}}\right)^{3}, \ldots\right\} .
\end{aligned}
$$

Proof. From Eq.(1.1), we see that

$$
x_{1}=\max \left\{\frac{A_{0}}{x_{0}}, x_{-2}\right\} .
$$

We consider the following two cases:

$$
\text { Case } \begin{aligned}
\left(a_{1}\right) \text { If } x_{-2} x_{0} & <A_{0} \text {, then } x_{1}=\frac{A_{0}}{x_{0}} \text { and } \\
x_{2} & =\max \left\{\frac{A_{1}}{x_{1}}, x_{-1}\right\}=\max \left\{\frac{A_{1} x_{0}}{A_{0}}, x_{-1}\right\} .
\end{aligned}
$$


$\left(a_{11}\right)$ If $x_{-1} A_{0}<A_{1} x_{0}$, then $x_{2}=\frac{A_{1} x_{0}}{A_{0}}$ and

$$
x_{3}=\max \left\{\frac{A_{2}}{x_{2}}, x_{0}\right\}=\max \left\{\frac{A_{0}^{2}}{A_{1} x_{0}}, x_{0}\right\} \text {. }
$$

$\left(a_{111}\right)$ If $A_{1} x_{0}^{2}<A_{0}^{2}$, then $x_{3}=\frac{A_{0}^{2}}{A_{1} x_{0}}$ and

$$
x_{4}=\max \left\{\frac{A_{3}}{x_{3}}, x_{1}\right\}=\max \left\{\frac{A_{1}^{2} x_{0}}{A_{0}^{2}}, \frac{A_{0}}{x_{0}}\right\},
$$

$\left(a_{1111}\right)$ If $A_{1}^{2} x_{0}^{2}<A_{0}^{3}$, then $x_{4}=\frac{A_{0}}{x_{0}}$ and

$$
\begin{gathered}
x_{5}=\max \left\{\frac{A_{4}}{x_{4}}, x_{2}\right\}=\max \left\{x_{0}, \frac{A_{1} x_{0}}{A_{0}}\right\}=\frac{A_{1} x_{0}}{A_{0}}, \\
x_{6}=\max \left\{\frac{A_{5}}{x_{5}}, x_{3}\right\}=\max \left\{\frac{A_{0}}{x_{0}}, \frac{A_{0}^{2}}{A_{1} x_{0}}\right\}=\frac{A_{0}}{x_{0}}, \\
x_{7}=\max \left\{\frac{A_{6}}{x_{6}}, x_{4}\right\}=\max \left\{x_{0}, \frac{A_{0}}{x_{0}}\right\}=\frac{A_{0}}{x_{0}}, \\
\left(\text { since } x_{0}<\frac{A_{0}^{2}}{x_{0} A_{1}}<\frac{A_{0} A_{1}}{x_{0} A_{1}}<\frac{A_{0}}{x_{0}}\right\}, \\
x_{8}=\max \left\{\frac{A_{7}}{x_{7}}, x_{5}\right\}=\max \left\{\frac{A_{1} x_{0}}{A_{0}}, \frac{A_{1} x_{0}}{A_{0}}\right\}=\frac{A_{1} x_{0}}{A_{0}}, \\
x_{9}=\max \left\{\frac{A_{8}}{x_{8}}, x_{6}\right\}=\max \left\{\frac{A_{0}^{2}}{A_{1} x_{0}}, \frac{A_{0}}{x_{0}}\right\}=\frac{A_{0}}{x_{0}},
\end{gathered}
$$

then the solution takes the form

$$
\left\{\frac{A_{0}}{x_{0}}, \frac{A_{1} x_{0}}{A_{0}}, \frac{A_{0}}{x_{0}}, \frac{A_{0}}{x_{0}}, \frac{A_{1} x_{0}}{A_{0}}, \frac{A_{0}}{x_{0}}, \frac{A_{0}}{x_{0}}, \ldots\right\},
$$

which is eventually periodic with period three.

$$
\begin{aligned}
& \left(a_{1112}\right) \text { If } A_{1}^{2} x_{0}^{2}>A_{0}^{3} \text {, then } x_{4}=\frac{A_{1}^{2} x_{0}}{A_{0}^{2}} \text { and } \\
& x_{5}=\max \left\{\frac{A_{4}}{x_{4}}, x_{2}\right\}=\max \left\{\frac{A_{0}^{3}}{A_{1}^{2} x_{0}}, \frac{A_{1} x_{0}}{A_{0}}\right\}=\frac{A_{1} x_{0}}{A_{0}}, \\
& \left(\text { since } A_{1}^{2} x_{0}^{2}>A_{0}^{3} \Rightarrow A_{1}^{3} x_{0}^{2}>A_{1} A_{0}^{3}>A_{0}^{4}\right),
\end{aligned}
$$




$$
\begin{aligned}
& x_{6}=\max \left\{\frac{A_{5}}{x_{5}}, x_{3}\right\}=\max \left\{\frac{A_{0}}{x_{0}}, \frac{A_{0}^{2}}{A_{1} x_{0}}\right\}=\frac{A_{0}}{x_{0}}, \\
& x_{7}=\max \left\{\frac{A_{6}}{x_{6}}, x_{4}\right\}=\max \left\{x_{0}, \frac{A_{1}^{2} x_{0}}{A_{0}^{2}}\right\}=\frac{A_{1}^{2} x_{0}}{A_{0}^{2}}, \\
& x_{8}=\max \left\{\frac{A_{7}}{x_{7}}, x_{5}\right\}=\max \left\{\frac{A_{0}^{2}}{A_{1} x_{0}}, \frac{A_{1} x_{0}}{A_{0}}\right\}=\frac{A_{1} x_{0}}{A_{0}}, \\
& x_{9}=\max \left\{\frac{A_{8}}{x_{8}}, x_{6}\right\}=\max \left\{\frac{A_{0}^{2}}{A_{1} x_{0}}, \frac{A_{0}}{x_{0}}\right\}=\frac{A_{0}}{x_{0}},
\end{aligned}
$$

and the solution has the following form which is period three solution

$$
\left\{\frac{A_{1}^{2} x_{0}}{A_{0}^{2}}, \frac{A_{1} x_{0}}{A_{0}}, \frac{A_{0}}{x_{0}}, \frac{A_{1}^{2} x_{0}}{A_{0}^{2}}, \frac{A_{1} x_{0}}{A_{0}}, \frac{A_{0}}{x_{0}}, \frac{A_{1}^{2} x_{0}}{A_{0}^{2}}, \ldots\right\} .
$$

$\left(a_{112}\right)$ If $A_{1} x_{0}^{2}>A_{0}^{2}$, then $x_{3}=x_{0}$ and

$$
\begin{aligned}
& x_{4}=\max \left\{\frac{A_{3}}{x_{3}}, x_{1}\right\}=\max \left\{\frac{A_{1}}{x_{0}}, \frac{A_{0}}{x_{0}}\right\}=\frac{A_{1}}{x_{0}}, \\
& x_{5}=\max \left\{\frac{A_{4}}{x_{4}}, x_{2}\right\}=\max \left\{\frac{A_{0} x_{0}}{A_{1}}, \frac{A_{1} x_{0}}{A_{0}}\right\}=\frac{A_{1} x_{0}}{A_{0}}, \\
& x_{6}=\max \left\{\frac{A_{5}}{x_{5}}, x_{3}\right\}=\max \left\{\frac{A_{0}}{x_{0}}, x_{0}\right\},
\end{aligned}
$$

$\left(a_{1121}\right)$ If $x_{0}^{2}>A_{0}$, then $x_{6}=x_{0}$ and

$$
\begin{aligned}
& x_{7}=\max \left\{\frac{A_{6}}{x_{6}}, x_{4}\right\}=\max \left\{\frac{A_{0}}{x_{0}}, \frac{A_{1}}{x_{0}}\right\}=\frac{A_{1}}{x_{0}}, \\
& x_{8}=\max \left\{\frac{A_{7}}{x_{7}}, x_{5}\right\}=\max \left\{x_{0}, \frac{A_{1} x_{0}}{A_{0}}\right\}=\frac{A_{1} x_{0}}{A_{0}}, \\
& x_{9}=\max \left\{\frac{A_{8}}{x_{8}}, x_{6}\right\}=\max \left\{\frac{A_{0}^{2}}{A_{1} x_{0}}, x_{0}\right\}=x_{0},
\end{aligned}
$$

then the solution is eventually periodic with period three and the solution has the following expression:

$$
\left\{\frac{A_{1} x_{0}}{A_{0}}, x_{0}, \frac{A_{1}}{x_{0}}, \frac{A_{1} x_{0}}{A_{0}}, x_{0}, \frac{A_{1}}{x_{0}}, \ldots\right\} .
$$


$\left(a_{1122}\right)$ If $x_{0}^{2}<A_{0}$, then $x_{6}=\frac{A_{0}}{x_{0}}$ and

$$
\begin{aligned}
& x_{7}=\max \left\{\frac{A_{6}}{x_{6}}, x_{4}\right\}=\max \left\{x_{0}, \frac{A_{1}}{x_{0}}\right\}=\frac{A_{1}}{x_{0}}, \\
& x_{8}=\max \left\{\frac{A_{7}}{x_{7}}, x_{5}\right\}=\max \left\{x_{0}, \frac{A_{1} x_{0}}{A_{0}}\right\}=\frac{A_{1} x_{0}}{A_{0}}, \\
& x_{9}=\max \left\{\frac{A_{8}}{x_{8}}, x_{6}\right\}=\max \left\{\frac{A_{0}^{2}}{A_{1} x_{0}}, \frac{A_{0}}{x_{0}}\right\}=\frac{A_{0}}{x_{0}},
\end{aligned}
$$

then this solution can be written as

$$
\left\{\frac{A_{1}}{x_{0}}, \frac{A_{1} x_{0}}{A_{0}}, \frac{A_{0}}{x_{0}}, \frac{A_{1}}{x_{0}}, \frac{A_{1} x_{0}}{A_{0}}, \frac{A_{0}}{x_{0}}, \frac{A_{1}}{x_{0}}, \ldots\right\},
$$

which is eventually periodic with period three.

$\left(a_{12}\right)$ If $x_{-1} A_{0}>A_{1} x_{0}$, then $x_{2}=x_{-1}$ and

$$
x_{3}=\max \left\{\frac{A_{2}}{x_{2}}, x_{0}\right\}=\max \left\{\frac{A_{0}}{x_{-1}}, x_{0}\right\} \text {. }
$$

$\left(a_{121}\right)$ If $x_{0}<\frac{A_{0}}{x_{-1}}$, then $x_{3}=\frac{A_{0}}{x_{-1}}$ and

$$
x_{4}=\max \left\{\frac{A_{3}}{x_{3}}, x_{1}\right\}=\max \left\{\frac{A_{1} x_{-1}}{A_{0}}, \frac{A_{0}}{x_{0}}\right\},
$$

$\left(a_{1211}\right)$ If $\frac{A_{1} x_{-1}}{A_{0}}<\frac{A_{0}}{x_{0}}$, then $x_{4}=\frac{A_{0}}{x_{0}}$ and

$$
\begin{gathered}
x_{5}=\max \left\{\frac{A_{4}}{x_{4}}, x_{2}\right\}=\max \left\{x_{0}, x_{-1}\right\}=x_{-1}, \\
\left(\text { since } A_{0} x_{-1}>A_{1} x_{0}>A_{0} x_{0}\right), \\
x_{6}=\max \left\{\frac{A_{5}}{x_{5}}, x_{3}\right\}=\max \left\{\frac{A_{1}}{x_{-1}}, \frac{A_{0}}{x_{-1}}\right\}=\frac{A_{1}}{x_{-1}}, \\
x_{7}=\max \left\{\frac{A_{6}}{x_{6}}, x_{4}\right\}=\max \left\{\frac{A_{0} x_{-1}}{A_{1}}, \frac{A_{0}}{x_{0}}\right\}=\frac{A_{0}}{x_{0}}, \\
\left(\operatorname{since} x_{0} x_{-1}<A_{0}<A_{1} \Rightarrow A_{0} x_{-1}<\frac{A_{1} A_{0}}{x_{0}}\right), \\
x_{8}=\max \left\{\frac{A_{7}}{x_{7}}, x_{5}\right\}=\max \left\{\frac{A_{1} x_{0}}{A_{0}}, x_{-1}\right\}=x_{-1}, \\
x_{9}=\max \left\{\frac{A_{8}}{x_{8}}, x_{6}\right\}=\max \left\{\frac{A_{0}}{x_{-1}}, \frac{A_{1}}{x_{-1}}\right\}=\frac{A_{1}}{x_{-1}},
\end{gathered}
$$


we obtain that the solution is eventually periodic with period three. It has the following form

$$
\left\{\frac{A_{0}}{x_{0}}, x_{-1}, \frac{A_{1}}{x_{-1}}, \frac{A_{0}}{x_{0}}, x_{-1}, \frac{A_{1}}{x_{-1}}, \ldots\right\} .
$$

$\left(a_{1212}\right)$ If $\frac{A_{1} x_{-1}}{A_{0}}>\frac{A_{0}}{x_{0}}$, then $x_{4}=\frac{A_{1} x_{-1}}{A_{0}}$ and

$$
\begin{gathered}
x_{5}=\max \left\{\frac{A_{4}}{x_{4}}, x_{2}\right\}=\max \left\{\frac{A_{0}^{2}}{A_{1} x_{-1}}, x_{-1}\right\}=x_{-1}, \\
\left(\text { since } x_{-1}>\frac{A_{1} x_{0}}{A_{0}} \& x_{0}>\frac{A_{0}^{2}}{A_{1} x_{-1}} \Rightarrow x_{-1}>\frac{A_{1} A_{0}^{2}}{A_{0} A_{1} x_{-1}}>\frac{A_{0}^{2}}{A_{1} x_{-1}}\right) \\
x_{6}=\max \left\{\frac{A_{5}}{x_{5}}, x_{3}\right\}=\max \left\{\frac{A_{1}}{x_{-1}}, \frac{A_{0}}{x_{-1}}\right\}=\frac{A_{1}}{x_{-1}}, \\
x_{7}=\max \left\{\frac{A_{6}}{x_{6}}, x_{4}\right\}=\max \left\{\frac{A_{0} x_{-1}}{A_{1}}, \frac{A_{1} x_{-1}}{A_{0}}\right\}=\frac{A_{1} x_{-1}}{A_{0}}, \\
x_{8}=\max \left\{\frac{A_{7}}{x_{7}}, x_{5}\right\}=\max \left\{\frac{A_{0}}{x_{-1}}, x_{-1}\right\}=x_{-1},
\end{gathered}
$$

(since $A_{1} x_{-1}^{2}>A_{0} A_{1}$ ),

$$
x_{9}=\max \left\{\frac{A_{8}}{x_{8}}, x_{6}\right\}=\max \left\{\frac{A_{0}}{x_{-1}}, \frac{A_{1}}{x_{-1}}\right\}=\frac{A_{1}}{x_{-1}},
$$

then the solution is eventually periodic with period three. Moreover, it has the following form:

$$
\left\{\frac{A_{1} x_{-1}}{A_{0}}, x_{-1}, \frac{A_{1}}{x_{-1}}, \frac{A_{1} x_{-1}}{A_{0}}, x_{-1}, \frac{A_{1}}{x_{-1}}, \ldots\right\} .
$$

$\left(a_{122}\right)$ If $A_{0}<x_{-1} x_{0}$, then $x_{3}=x_{0}$ and

$$
\begin{aligned}
x_{4} & =\max \left\{\frac{A_{3}}{x_{3}}, x_{1}\right\}=\max \left\{\frac{A_{1}}{x_{0}}, \frac{A_{0}}{x_{0}}\right\}=\frac{A_{1}}{x_{0}}, \\
x_{5} & =\max \left\{\frac{A_{4}}{x_{4}}, x_{2}\right\}=\max \left\{\frac{A_{0} x_{0}}{A_{1}}, x_{-1}\right\}=x_{-1}, \\
& \left(\text { since } x_{-1}>\frac{A_{1} x_{0}}{A_{0}}>\frac{A_{0} x_{0}}{A_{1}}\right), \\
x_{6} & =\max \left\{\frac{A_{5}}{x_{5}}, x_{3}\right\}=\max \left\{\frac{A_{1}}{x_{-1}}, x_{0}\right\} .
\end{aligned}
$$

$\left(a_{1221}\right)$ If $A_{1}<x_{0} x_{-1}$, then $x_{6}=x_{0}$ and

$$
x_{7}=\max \left\{\frac{A_{6}}{x_{6}}, x_{4}\right\}=\max \left\{\frac{A_{0}}{x_{0}}, \frac{A_{1}}{x_{0}}\right\}=\frac{A_{1}}{x_{0}},
$$




$$
\begin{gathered}
x_{8}=\max \left\{\frac{A_{7}}{x_{7}}, x_{5}\right\}=\max \left\{x_{0}, x_{-1}\right\}=x_{-1}, \\
\left(\text { since } x_{-1}>\frac{A_{1} x_{0}}{A_{0}}>\frac{A_{0} x_{0}}{A_{0}}=x_{0}\right), \\
x_{9}=\max \left\{\frac{A_{8}}{x_{8}}, x_{6}\right\}=\max \left\{\frac{A_{0}}{x_{-1}}, x_{0}\right\}=x_{0},
\end{gathered}
$$

the developed solution has the following form

$$
\left\{x_{-1}, x_{0}, \frac{A_{1}}{x_{0}}, x_{-1}, x_{0}, \frac{A_{1}}{x_{0}}, \ldots\right\} .
$$

$\left(a_{1222}\right)$ If $A_{1}>x_{0} x_{-1}$, then $x_{6}=\frac{A_{1}}{x_{-1}}$ and

$$
\begin{aligned}
x_{7}=\max \left\{\frac{A_{6}}{x_{6}}, x_{4}\right\}=\max \left\{\frac{A_{0} x_{-1}}{A_{1}}, \frac{A_{1}}{x_{0}}\right\}=\frac{A_{1}}{x_{0}}, \\
\left(\text { since } A_{1}>x_{0} x_{-1} \Rightarrow A_{1}^{2}>A_{1} A_{0}>A_{0} x_{0} x_{-1}\right), \\
x_{8}=\max \left\{\frac{A_{7}}{x_{7}}, x_{5}\right\}=\max \left\{x_{0}, x_{-1}\right\}=x_{-1}, \\
\left(\text { since } x_{-1}>\frac{A_{1} x_{0}}{A_{0}}>\frac{A_{0} x_{0}}{A_{0}}=x_{0}\right), \\
x_{9}=\max \left\{\frac{A_{8}}{x_{8}}, x_{6}\right\}=\max \left\{\frac{A_{0}}{x_{-1}}, \frac{A_{1}}{x_{-1}}\right\}=\frac{A_{1}}{x_{-1}},
\end{aligned}
$$

we get the solution which is eventually periodic with period three. It has the following form

$$
\left\{\frac{A_{1}}{x_{0}}, x_{-1}, \frac{A_{1}}{x_{-1}}, \frac{A_{1}}{x_{0}}, x_{-1}, \frac{A_{1}}{x_{-1}}, \ldots\right\} .
$$

Case $\left(a_{2}\right)$ If $x_{-2} x_{0}>A_{0}$, then $x_{1}=x_{-2}$ and

$$
x_{2}=\max \left\{\frac{A_{1}}{x_{1}}, x_{-1}\right\}=\max \left\{\frac{A_{1}}{x_{-2}}, x_{-1}\right\} .
$$

$\left(a_{21}\right)$ If $x_{-1} x_{-2}<A_{1}$, then $x_{2}=\frac{A_{1}}{x_{-2}}$ and

$$
x_{3}=\max \left\{\frac{A_{2}}{x_{2}}, x_{0}\right\}=\max \left\{\frac{A_{0} x_{-2}}{A_{1}}, x_{0}\right\} .
$$

$\left(a_{211}\right)$ If $A_{1} x_{0}>A_{0} x_{-2}$, then $x_{3}=x_{0}$ and

$$
x_{4}=\max \left\{\frac{A_{3}}{x_{3}}, x_{1}\right\}=\max \left\{\frac{A_{1}}{x_{0}}, x_{-2}\right\} .
$$


$\left(a_{2111}\right)$ If $A_{1}>x_{0} x_{-2}$, then $x_{4}=\frac{A_{1}}{x_{0}}$ and

$$
x_{5}=\max \left\{\frac{A_{4}}{x_{4}}, x_{2}\right\}=\max \left\{\frac{A_{0} x_{0}}{A_{1}}, \frac{A_{1}}{x_{-2}}\right\}=\frac{A_{1}}{x_{-2}},
$$

(since $A_{1}>x_{0} x_{-2} \Rightarrow>A_{1}^{2}>A_{1} x_{0} x_{-2}>A_{0} x_{0} x_{-2}$ ),

$$
x_{6}=\max \left\{\frac{A_{5}}{x_{5}}, x_{3}\right\}=\max \left\{x_{-2}, x_{0}\right\} .
$$

$\left(a_{21111}\right)$ If $x_{0}<x_{-2}$, then $x_{6}=x_{-2}$ and

$$
x_{7}=\max \left\{\frac{A_{6}}{x_{6}}, x_{4}\right\}=\max \left\{\frac{A_{0}}{x_{-2}}, \frac{A_{1}}{x_{0}}\right\}=\frac{A_{1}}{x_{0}},
$$

(since $A_{1} x_{-2}>A_{1} x_{0}>A_{0} x_{0}$ ),

$$
\begin{aligned}
& x_{8}=\max \left\{\frac{A_{7}}{x_{7}}, x_{5}\right\}=\max \left\{\frac{A_{0} x_{0}}{A_{1}}, \frac{A_{1}}{x_{-2}}\right\}=\frac{A_{1}}{x_{-2}}, \\
& x_{9}=\max \left\{\frac{A_{8}}{x_{8}}, x_{6}\right\}=\max \left\{\frac{A_{0} x_{-2}}{A_{1}}, x_{-2}\right\}=x_{-2},
\end{aligned}
$$

the solution of the form

$$
\left\{x_{-2}, \frac{A_{1}}{x_{0}}, \frac{A_{1}}{x_{-2}}, x_{-2}, \frac{A_{1}}{x_{0}}, \frac{A_{1}}{x_{-2}}, x_{-2}, \ldots\right\},
$$

is eventually periodic with period three.

$\left(a_{21112}\right)$ If $x_{0}>x_{-2}$, then $x_{6}=x_{0}$ and

$$
\begin{aligned}
& x_{7}=\max \left\{\frac{A_{6}}{x_{6}}, x_{4}\right\}=\max \left\{\frac{A_{0}}{x_{0}}, \frac{A_{1}}{x_{0}}\right\}=\frac{A_{1}}{x_{0}}, \\
& x_{8}=\max \left\{\frac{A_{7}}{x_{7}}, x_{5}\right\}=\max \left\{x_{0}, \frac{A_{1}}{x_{-2}}\right\}=\frac{A_{1}}{x_{-2}}, \\
& x_{9}=\max \left\{\frac{A_{8}}{x_{8}}, x_{6}\right\}=\max \left\{\frac{A_{0} x_{-2}}{A_{1}}, x_{0}\right\}=x_{0},
\end{aligned}
$$

this shows that the solution

$$
\left\{x_{0}, \frac{A_{1}}{x_{0}}, \frac{A_{1}}{x_{-2}}, x_{0}, \frac{A_{1}}{x_{0}}, \frac{A_{1}}{x_{-2}}, x_{0}, \ldots\right\},
$$

is just eventually periodic with period three.

$\left(a_{2112}\right)$ If $A_{1}<x_{0} x_{-2}$, then $x_{4}=x_{-2}$ and

$$
\begin{aligned}
& x_{5}=\max \left\{\frac{A_{4}}{x_{4}}, x_{2}\right\}=\max \left\{\frac{A_{0}}{x_{-2}}, \frac{A_{1}}{x_{-2}}\right\}=\frac{A_{1}}{x_{-2}}, \\
& x_{6}=\max \left\{\frac{A_{5}}{x_{5}}, x_{3}\right\}=\max \left\{x_{-2}, x_{0}\right\} .
\end{aligned}
$$


$\left(a_{21121}\right)$ If $x_{0}<x_{-2}$, then $x_{6}=x_{-2}$ and

$$
\begin{aligned}
x_{7} & =\max \left\{\frac{A_{6}}{x_{6}}, x_{4}\right\}=\max \left\{\frac{A_{0}}{x_{-2}}, x_{-2}\right\}=x_{-2}, \\
\text { (since } x_{-2} & \left.>\frac{A_{1}}{x_{0}}>\frac{A_{1}}{x_{-2}}>\frac{A_{0}}{x_{-2}}\right), \\
x_{8} & =\max \left\{\frac{A_{7}}{x_{7}}, x_{5}\right\}=\max \left\{\frac{A_{1}}{x_{-2}}, \frac{A_{1}}{x_{-2}}\right\}=\frac{A_{1}}{x_{-2}}, \\
x_{9} & =\max \left\{\frac{A_{8}}{x_{8}}, x_{6}\right\}=\max \left\{\frac{A_{0} x_{-2}}{A_{1}}, x_{-2}\right\}=x_{-2},
\end{aligned}
$$

this gives the solution

$$
\left\{x_{-2}, \frac{A_{1}}{x_{-2}}, x_{-2}, x_{-2}, \frac{A_{1}}{x_{-2}}, x_{-2}, x_{-2}, \ldots\right\} .
$$

$\left(a_{21122}\right)$ If $x_{0}>x_{-2}$, then $x_{6}=x_{0}$ and

$$
\begin{aligned}
& x_{7}=\max \left\{\frac{A_{6}}{x_{6}}, x_{4}\right\}=\max \left\{\frac{A_{0}}{x_{0}}, x_{-2}\right\}=x_{-2}, \\
& x_{8}=\max \left\{\frac{A_{7}}{x_{7}}, x_{5}\right\}=\max \left\{\frac{A_{1}}{x_{-2}}, \frac{A_{1}}{x_{-2}}\right\}=\frac{A_{1}}{x_{-2}}, \\
& x_{9}=\max \left\{\frac{A_{8}}{x_{8}}, x_{6}\right\}=\max \left\{\frac{A_{0} x_{-2}}{A_{1}}, x_{0}\right\}=x_{0},
\end{aligned}
$$

then the solution is eventually periodic with period three, moreover, it has the following form

$$
\left\{x_{0}, x_{-2}, \frac{A_{1}}{x_{-2}}, x_{0}, x_{-2}, \frac{A_{1}}{x_{-2}}, x_{0}, \ldots\right\}
$$

$\left(a_{212}\right)$ If $A_{1} x_{0}<A_{0} x_{-2}$, then $x_{3}=\frac{A_{0} x_{-2}}{A_{1}}$ and

$$
x_{4}=\max \left\{\frac{A_{3}}{x_{3}}, x_{1}\right\}=\max \left\{\frac{A_{1}^{2}}{A_{0} x_{-2}}, x_{-2}\right\} .
$$

$\left(a_{2121}\right)$ If $A_{1}^{2}<A_{0} x_{-2}^{2}$, then $x_{4}=x_{-2}$ and

$$
\begin{aligned}
& x_{5}=\max \left\{\frac{A_{4}}{x_{4}}, x_{2}\right\}=\max \left\{\frac{A_{0}}{x_{-2}}, \frac{A_{0} x_{-2}}{A_{1}}\right\}=\frac{A_{0} x_{-2}}{A_{1}}, \\
& x_{6}=\max \left\{\frac{A_{5}}{x_{5}}, x_{3}\right\}=\max \left\{\frac{A_{1}^{2}}{A_{0} x_{-2}}, \frac{A_{0} x_{-2}}{A_{1}}\right\} .
\end{aligned}
$$


$\left(a_{21211}\right)$ If $A_{1}^{3}<A_{0}^{2} x_{-2}^{2}$, then $x_{6}=\frac{A_{0} x_{-2}}{A_{1}}$ and

$$
x_{7}=\max \left\{\frac{A_{6}}{x_{6}}, x_{4}\right\}=\max \left\{\frac{A_{1}}{x_{-2}}, x_{-2}\right\}=x_{-2},
$$

(since $A_{0} x_{-2}^{2}>A_{1}^{2}>A_{0} A_{1}$ ),

$$
\begin{aligned}
& x_{8}=\max \left\{\frac{A_{7}}{x_{7}}, x_{5}\right\}=\max \left\{\frac{A_{1}}{x_{-2}}, \frac{A_{0} x_{-2}}{A_{1}}\right\}=\frac{A_{0} x_{-2}}{A_{1}}, \\
& x_{9}=\max \left\{\frac{A_{8}}{x_{8}}, x_{6}\right\}=\max \left\{\frac{A_{1}}{x_{-2}}, \frac{A_{0} x_{-2}}{A_{1}}\right\}=\frac{A_{0} x_{-2}}{A_{1}},
\end{aligned}
$$

and the solution has the following expression

$$
\left\{x_{-2}, \frac{A_{0} x_{-2}}{A_{1}}, \frac{A_{0} x_{-2}}{A_{1}}, x_{-2}, \frac{A_{0} x_{-2}}{A_{1}}, \frac{A_{0} x_{-2}}{A_{1}}, x_{-2}, \ldots\right\} .
$$

$\left(a_{21212}\right)$ If $A_{1}^{3}>A_{0}^{2} x_{-2}^{2}$, then $x_{6}=\frac{A_{1}^{2}}{A_{0} x_{-2}}$ and

$$
\begin{aligned}
& x_{7}=\max \left\{\frac{A_{6}}{x_{6}}, x_{4}\right\}=\max \left\{\frac{A_{0}^{2}}{A_{1}^{2}} x_{-2}, x_{-2}\right\}=x_{-2}, \\
& x_{8}=\max \left\{\frac{A_{7}}{x_{7}}, x_{5}\right\}=\max \left\{\frac{A_{1}}{x_{-2}}, \frac{A_{0} x_{-2}}{A_{1}}\right\}=\frac{A_{0} x_{-2}}{A_{1}}, \\
& x_{9}=\max \left\{\frac{A_{8}}{x_{8}}, x_{6}\right\}=\max \left\{\frac{A_{1}}{x_{-2}}, \frac{A_{1}^{2}}{A_{0} x_{-2}}\right\}=\frac{A_{1}^{2}}{A_{0} x_{-2}},
\end{aligned}
$$

also the the solution has the following period three as follows

$$
\left\{x_{-2}, \frac{A_{0} x_{-2}}{A_{1}}, \frac{A_{1}^{2}}{A_{0} x_{-2}}, x_{-2}, \frac{A_{0} x_{-2}}{A_{1}}, \frac{A_{1}^{2}}{A_{0} x_{-2}}, x_{-2}, \ldots\right\} \text {. }
$$

$\left(a_{2122}\right)$ If $A_{1}^{2}>A_{0} x_{-2}^{2}$, then $x_{4}=\frac{A_{1}^{2}}{A_{0} x_{-2}}$ and

$$
x_{5}=\max \left\{\frac{A_{4}}{x_{4}}, x_{2}\right\}=\max \left\{\frac{A_{0}^{2} x_{-2}}{A_{1}^{2}}, \frac{A_{1}}{x_{-2}}\right\} \text {. }
$$

Case (i) If we take $x_{5}=\frac{A_{1}}{x_{-2}}$. Then the solution has the following form:

$$
\left\{\frac{A_{1}}{x_{-2}}, x_{-2}, \frac{A_{1}^{2}}{A_{0} x_{-2}}, \frac{A_{1}}{x_{-2}}, x_{-2}, \frac{A_{1}^{2}}{A_{0} x_{-2}}, \frac{A_{1}}{x_{-2}}, x_{-2}, \ldots\right\} .
$$


Case (ii) If $x_{5}=\frac{A_{0}^{2} x_{-2}}{A_{1}^{2}}$, then the solution will be periodic with period three or being in the following form

$$
\begin{aligned}
\left\{x_{2 n-1}\right\}_{n=1}^{\infty} & =\left\{x_{-2}, x_{-2}\left(\frac{A_{0}}{A_{1}}\right), x_{-2}\left(\frac{A_{0}}{A_{1}}\right)^{2}, x_{-2}\left(\frac{A_{0}}{A_{1}}\right)^{3}, \ldots\right\}, \\
\left\{x_{2 n}\right\}_{n=1}^{\infty} & =\left\{\frac{A_{1}}{x_{-2}}, \frac{A_{1}}{x_{-2}}\left(\frac{A_{1}}{A_{0}}\right), \frac{A_{1}}{x_{-2}}\left(\frac{A_{1}}{A_{0}}\right)^{2}, \frac{A_{1}}{x_{-2}}\left(\frac{A_{1}}{A_{0}}\right)^{3}, \ldots\right\} .
\end{aligned}
$$

which is unboundedness solution.

$\left(a_{22}\right)$ If $x_{-1} x_{-2}>A_{1}$, then $x_{2}=x_{-1}$ and

$$
x_{3}=\max \left\{\frac{A_{2}}{x_{2}}, x_{0}\right\}=\max \left\{\frac{A_{0}}{x_{-1}}, x_{0}\right\} .
$$

$\left(a_{221}\right)$ If $x_{0} x_{-1}<A_{0}$, then $x_{3}=\frac{A_{0}}{x_{-1}}$ and

$$
x_{4}=\max \left\{\frac{A_{3}}{x_{3}}, x_{1}\right\}=\max \left\{\frac{A_{1} x_{-1}}{A_{0}}, x_{-2}\right\} .
$$

$\left(a_{2211}\right)$ If $A_{0} x_{-2}<A_{1} x_{-1}$, then $x_{4}=\frac{A_{1} x_{-1}}{A_{0}}$ and

$$
x_{5}=\max \left\{\frac{A_{4}}{x_{4}}, x_{2}\right\}=\max \left\{\frac{A_{0}^{2}}{A_{1} x_{-1}}, x_{-1}\right\}=x_{-1},
$$

(since $x_{-1}>\frac{A_{1}}{x_{-2}} \& A_{1} x_{-1}>A_{0} x_{-2} \Rightarrow A_{1} x_{-1}^{2}>A_{0} A_{1}>A_{0}^{2}$ ),

$$
\begin{aligned}
& x_{6}=\max \left\{\frac{A_{5}}{x_{5}}, x_{3}\right\}=\max \left\{\frac{A_{1}}{x_{-1}}, \frac{A_{0}}{x_{-1}}\right\}=\frac{A_{1}}{x_{-1}}, \\
& x_{7}=\max \left\{\frac{A_{6}}{x_{6}}, x_{4}\right\}=\max \left\{\frac{A_{0} x_{-1}}{A_{1}}, \frac{A_{1} x_{-1}}{A_{0}}\right\}=\frac{A_{1} x_{-1}}{A_{0}}, \\
& x_{8}=\max \left\{\frac{A_{7}}{x_{7}}, x_{5}\right\}=\max \left\{\frac{A_{0}}{x_{-1}}, x_{-1}\right\}=x_{-1},
\end{aligned}
$$

(since $A_{1} x_{-1}^{2}>A_{1} A_{0}$ ),

$$
x_{9}=\max \left\{\frac{A_{8}}{x_{8}}, x_{6}\right\}=\max \left\{\frac{A_{0}}{x_{-1}}, \frac{A_{1}}{x_{-1}}\right\}=\frac{A_{1}}{x_{-1}} .
$$

Obviously, the solution is eventually periodic with period three, which can be expressed in the form

$$
\left\{x_{-1}, \frac{A_{1}}{x_{-1}}, \frac{A_{1} x_{-1}}{A_{0}}, x_{-1}, \frac{A_{1}}{x_{-1}}, \frac{A_{1} x_{-1}}{A_{0}}, \ldots\right\} .
$$


$\left(a_{2212}\right)$ If $A_{0} x_{-2}>A_{1} x_{-1}$, then $x_{4}=x_{-2}$ and

$$
\begin{gathered}
x_{5}=\max \left\{\frac{A_{4}}{x_{4}}, x_{2}\right\}=\max \left\{\frac{A_{0}}{x_{-2}}, x_{-1}\right\}=x_{-1}, \\
x_{6}=\max \left\{\frac{A_{5}}{x_{5}}, x_{3}\right\}=\max \left\{\frac{A_{1}}{x_{-1}}, \frac{A_{0}}{x_{-1}}\right\}=\frac{A_{1}}{x_{-1}}, \\
x_{7}=\max \left\{\frac{A_{6}}{x_{6}}, x_{4}\right\}=\max \left\{\frac{A_{0} x_{-1}}{A_{1}}, x_{-2}\right\}=x_{-2}, \\
\text { since } \left.x_{-2}>\frac{A_{1} x_{-1}}{A_{0}}>\frac{A_{0} x_{-1}}{A_{1}}\right), \\
x_{8}=\max \left\{\frac{A_{7}}{x_{7}}, x_{5}\right\}=\max \left\{\frac{A_{1}}{x_{-2}}, x_{-1}\right\}=x_{-1}, \\
x_{9}=\max \left\{\frac{A_{8}}{x_{8}}, x_{6}\right\}=\max \left\{\frac{A_{0}}{x_{-1}}, \frac{A_{1}}{x_{-1}}\right\}=\frac{A_{1}}{x_{-1}},
\end{gathered}
$$

this provides an eventually periodic with period three, which takes the form

$$
\left\{\frac{A_{1}}{x_{-1}}, x_{-2}, x_{-1}, \frac{A_{1}}{x_{-1}}, x_{-2}, x_{-1}, \ldots\right\} .
$$

$\left(a_{222}\right)$ If $A_{0}<x_{-1} x_{0}$, then $x_{3}=x_{0}$ and

$$
x_{4}=\max \left\{\frac{A_{3}}{x_{3}}, x_{1}\right\}=\max \left\{\frac{A_{1}}{x_{0}}, x_{-2}\right\} .
$$

$\left(a_{2221}\right)$ If $A_{1}<x_{-2} x_{0}$, then $x_{4}=x_{-2}$ and

$$
\begin{aligned}
& x_{5}=\max \left\{\frac{A_{4}}{x_{4}}, x_{2}\right\}=\max \left\{\frac{A_{0}}{x_{-2}}, x_{-1}\right\}=x_{-1}, \\
& x_{6}=\max \left\{\frac{A_{5}}{x_{5}}, x_{3}\right\}=\max \left\{\frac{A_{1}}{x_{-1}}, x_{0}\right\} .
\end{aligned}
$$

$\left(a_{22211}\right)$ If $\frac{A_{1}}{x_{-1}}<x_{0}$, then $x_{6}=x_{0}$ and

$$
\begin{aligned}
& x_{7}=\max \left\{\frac{A_{6}}{x_{6}}, x_{4}\right\}=\max \left\{\frac{A_{0}}{x_{0}}, x_{-2}\right\}=x_{-2}, \\
& x_{8}=\max \left\{\frac{A_{7}}{x_{7}}, x_{5}\right\}=\max \left\{\frac{A_{1}}{x_{-2}}, x_{-1}\right\}=x_{-1}, \\
& x_{9}=\max \left\{\frac{A_{8}}{x_{8}}, x_{6}\right\}=\max \left\{\frac{A_{0}}{x_{-1}}, x_{0}\right\}=x_{0},
\end{aligned}
$$

and the solution has the following form

$$
\left\{x_{-2}, x_{-1}, x_{0}, x_{-2}, x_{-1}, x_{0}, \ldots\right\} .
$$


$\left(a_{22212}\right)$ If $\frac{A_{1}}{x_{-1}}<x_{0}$, then $x_{6}=\frac{A_{1}}{x_{-1}}$ and

$$
\begin{aligned}
& x_{7}= \max \left\{\frac{A_{6}}{x_{6}}, x_{4}\right\}=\max \left\{\frac{A_{0} x_{-1}}{A_{1}}, x_{-2}\right\}=x_{-2}, \\
&\left(\text { since } x_{-2} x_{-1}>A_{1}>x_{0} x_{-1} \Rightarrow x_{-2}>x_{-1} \Rightarrow A_{1} x_{-2}>A_{1} x_{-1}>A_{0} x_{-1}\right), \\
& x_{8}= \max \left\{\frac{A_{7}}{x_{7}}, x_{5}\right\}=\max \left\{\frac{A_{1}}{x_{-2}}, x_{-1}\right\}=x_{-1}, \\
& x_{9}=\max \left\{\frac{A_{8}}{x_{8}}, x_{6}\right\}=\max \left\{\frac{A_{0}}{x_{-1}}, \frac{A_{1}}{x_{-1}}\right\}=\frac{A_{1}}{x_{-1}},
\end{aligned}
$$

we obtain that the solution is eventually periodic with period three. It has the following form

$$
\left\{x_{-2}, x_{-1}, \frac{A_{1}}{x_{-1}}, x_{-2}, x_{-1}, \frac{A_{1}}{x_{-1}}, \ldots\right\}
$$

$\left(a_{2222}\right)$ If $A_{1}>x_{-2} x_{0}$, then $x_{4}=\frac{A_{1}}{x_{0}}$ and

$$
x_{5}=\max \left\{\frac{A_{4}}{x_{4}}, x_{2}\right\}=\max \left\{\frac{A_{0} x_{0}}{A_{1}}, x_{-1}\right\}=x_{-1},
$$

(since $x_{-2} x_{-1}>A_{1}>x_{0} x_{-2} \Rightarrow x_{-1}>x_{0} \Rightarrow A_{1} x_{-1}>A_{1} x_{0}>A_{0} x_{0}$ ),

$$
x_{6}=\max \left\{\frac{A_{5}}{x_{5}}, x_{3}\right\}=\max \left\{\frac{A_{1}}{x_{-1}}, x_{-1}\right\} \text {. }
$$

$\left(a_{22221}\right)$ If $\frac{A_{1}}{x_{-1}}>x_{-1}$, then $x_{6}=\frac{A_{1}}{x_{-1}}$ and

$$
\begin{aligned}
x_{7}=\max \left\{\frac{A_{6}}{x_{6}}, x_{4}\right\}=\max \left\{\frac{A_{0} x_{-1}}{A_{1}}, \frac{A_{1}}{x_{0}}\right\}=\frac{A_{1}}{x_{0}}, \\
\left(\text { since } A_{1}>x_{-1}^{2}>x_{-1} x_{0} \Rightarrow A_{1}^{2} x_{-1}>A_{1} x_{-1} x_{0}>A_{0} x_{-1} x_{0}\right), \\
x_{8}=\max \left\{\frac{A_{7}}{x_{7}}, x_{5}\right\}=\max \left\{x_{0}, x_{-1}\right\}=x_{-1}, \\
x_{9}=\max \left\{\frac{A_{8}}{x_{8}}, x_{6}\right\}=\max \left\{\frac{A_{0}}{x_{-1}}, \frac{A_{1}}{x_{-1}}\right\}=\frac{A_{1}}{x_{-1}} .
\end{aligned}
$$

Then the solution is eventually periodic with period three and has the following form

$$
\left\{\frac{A_{1}}{x_{0}}, x_{-1}, \frac{A_{1}}{x_{-1}}, \frac{A_{1}}{x_{0}}, x_{-1}, \frac{A_{1}}{x_{-1}}, \ldots\right\} .
$$


$\left(a_{22222}\right)$ If $\frac{A_{1}}{x_{-1}}<x_{-1}$, then $x_{6}=x_{-1}$ and

$$
\begin{aligned}
& x_{7}=\max \left\{\frac{A_{6}}{x_{6}}, x_{4}\right\}=\max \left\{\frac{A_{0}}{x_{-1}}, \frac{A_{1}}{x_{0}}\right\}=\frac{A_{1}}{x_{0}}, \\
& x_{8}=\max \left\{\frac{A_{7}}{x_{7}}, x_{5}\right\}=\max \left\{x_{0}, x_{-1}\right\}=x_{-1}, \\
& x_{9}=\max \left\{\frac{A_{8}}{x_{8}}, x_{6}\right\}=\max \left\{\frac{A_{0}}{x_{-1}}, x_{-1}\right\}=x_{-1},
\end{aligned}
$$

we obtain that the solution is eventually periodic with period three and takes the form

$$
\left\{x_{-1}, x_{-1}, \frac{A_{1}}{x_{0}}, x_{-1}, x_{-1}, \frac{A_{1}}{x_{0}}, \ldots\right\} .
$$

\section{REFERENCES}

[1] A. M. Amleh, J. Hoag, and G. Ladas, "A difference equation with eventually periodic solutions," Computers and Mathematics with Applications, vol. 36, pp. 401-404, 1998, doi: 10.1016/S08981221(98)80040-0.

[2] W. J. Briden, E. A. Grove, G. Ladas, and C. M. Kent, "Eventually periodic solutions of $x_{n+1}=$ $\max \left\{\frac{1}{x_{n}}, \frac{a_{n}}{x_{n-1}}\right\}, "$ Communications on Applied Nonlinear Analysis, vol. 6, pp. 31-34, 1999.

[3] C. Cinar, S. Stevic, and I. Yalcinkaya, "On positive solutions of a reciprocal difference equation with minimum," Journal of Applied Mathematics and Computing, vol. 17, no. 1-2, pp. 307-314, 2005, doi: 10.1007/BF02936057.

[4] C. Cinar and I. Yalcinkaya, "On the positive solutions of the difference equation $x_{n+1}=$ $\max \left\{\frac{A}{x_{n}^{2}}, \frac{B x_{n-1}}{x_{n} x_{n-2}^{2}}\right\}, "$ International Journal of Contemporary Mathematical Sciences, vol. 1, no. 10, pp. 489-494, 2006.

[5] H. El-Metwally, E. M. Elabbasy, and E. M. Elsayed, "The periodicity character of a difference equation,” International Journal of Nonlinear Science, vol. 8, no. 3, pp. 291-299, 2009.

[6] E. M. Elabbasy, H. El-Metwally, and E. M. Elsayed, "On the periodic nature of some max-type difference equations," International Journal of Mathematics and Mathematical Sciences, vol. 14, pp. 2227-2239, 2005, doi: 10.1155/IJMMS.2005.2227.

[7] E. M. Elabbasy and E. M. Elsayed, "On the solution of recursive sequence $x_{n+1}=$ $\max \left\{x_{n-2}, \frac{1}{x_{n-2}}\right\}, "$ Fasciculi Mathematici, vol. 41, pp. 55-63, 2009.

[8] E. M. Elsayed, "Solution and attractivity for a rational recursive sequence," Discrete Dynamics in Nature and Society, vol. 2011, pp. 1-17, 2011, Article ID 982309, doi: 10.1155/2011/982309.

[9] E. M. Elsayed, "Solutions of rational difference system of order two," Mathematical and Computer Modelling, vol. 55, pp. 378-384, 2012, doi: 10.1016/j.mcm.2011.08.012.

[10] E. M. Elsayed, B. Iricanin, and S. Stevic, "On the max-type equation," Ars Combinatoria, vol. 95, pp. 187-192, 2010.

[11] E. M. Elsayed and B. D. Iricanin, "On a max-type and a min-type difference equation,” Applied Mathematics and Computations, vol. 215, pp. 608-614, 2009, doi: 10.1016/j.amc.2009.05.045. 
[12] E. M. Elsayed and S. Stevic, "On the max-type equation $x_{n+1}=\max \left\{\frac{A}{x_{n}}, x_{n-2}\right\}$," Nonlinear Analysis: Theory, Methods \& Applications, vol. 71, no. 3-4, pp. 910-922, 2009, doi: /10.1016/j.na.2008.11.016.

[13] A. Gelisken, C. Cinar, and I. Yalcinkaya, "On the periodicity of a difference equation with maximum," Nonlinear Dynamics, vol. 2008, pp. 1-11, 2008, Article ID 820629, doi: $10.1155 / 2008 / 820629$.

[14] E. A. Grove and G. Ladas, Periodicities in nonlinear difference equations. Chapman \& Hall, CRC Press, 2005.

[15] B. D. Iricanin and E. M. Elsayed, "On the max-type difference equation $x_{n+1}=$ $\max \left\{A / x_{n}, x_{n-2}\right\}, "$ Discrete Dynamics in Nature and Society, vol. 2010, pp. 1-13, 2010, Article ID 675413, doi: 10.1155/2010/675413.

[16] W. Liu and S. Stevic, "Global attractivity of a family of nonautonomous max-type difference equations," Applied Mathematics and Computation, vol. 218, pp. 6297-6303, 2012, doi: 10.1016/j.amc.2011.11.108.

[17] A. D. Myshkis, "On some problems of the theory of differential equations with deviating argument," Uspekhi Matematicheskikh Nauk, vol. 32, no. 194, pp. 173-202, 1977.

[18] E. P. Popov, Automatic regulation and control. Nauka, Moscow, Russia (in Russian), 1966.

[19] D. Simsek, C. Cinar, and I. Yalcinkaya, "On the solutions of the difference equation $x_{n+1}=$ $\max \left\{x_{n-1}, \frac{1}{x_{n-1}}\right\},$, International Journal of Contemporary Mathematical Sciences, vol. 1, no. 10 , pp. $481-487,2006$.

[20] D. Simsek, B. Demir, and C. Cinar, "On the solutions of the system of difference equations $x_{n+1}=\max \left\{\frac{A}{x_{n}}, \frac{y_{n}}{x_{n}}\right\}, y_{n+1}=\max \left\{\frac{A}{y_{n}}, \frac{x_{n}}{y_{n}}\right\},, "$ Discrete Dynamics in Nature and Society, vol. 2009, pp. 1-11, 2009, Article ID 325296, doi: 10.1155/2009/325296.

[21] S. Stevic, "On the recursive sequence $x_{n+1}=\max \left\{c, \frac{x_{n}^{p}}{x_{n-1}^{p}}\right\}$," Applied Mathematics Letters, vol. 21, no. 8, pp. 791-796, 2008.

[22] F. Sun, "On the asymptotic behavior of a difference equation with maximum," Discrete Dynamics in Nature and Society, vol. 2008, pp. 1-6, 2008, Article ID 243291, doi: 10.1155/2008/243291.

[23] N. Touafek and E. M. Elsayed, "On the solutions of systems of rational difference equations," Mathematical and Computer Modelling, vol. 55, pp. 1987-1997, 2012, doi: 10.1016/j.mcm.2011.11.058.

[24] N. Touafek and Y. Halim, "On max type difference equations: expressions of solutions," International Journal of Nonlinear Science, vol. 11, no. 4, pp. 396-402, 2011.

[25] I. Yalcinkaya, C. Cinar, and A. Gelisken, "On the recursive sequence $x_{n}+1=$ $\max \left\{x_{n}, A\right\} / x_{n}^{2} x_{n-1}, "$ Discrete Dynamics in Nature and Society, vol. 2010, pp. 1-13, 2010, Article ID 583230, doi: 10.1155/2010/583230.

[26] I. Yalcinkaya, B. D. Iricanin, and C. Cinar, "On a max-type difference equation," Discrete Dynamics in Nature and Society, vol. 2007, pp. 1-11, 2007, Article ID 47264, doi: 10.1155/2007/47264.

[27] X. Yang and X. Liao, "On a difference equation with maximum," Applied Mathematics and Computation, vol. 181, pp. 1-5, 2006.

\section{Author's address}

\section{E. M. Elsayed}

King AbdulAziz University, Faculty of Science, Mathematics Department, P. O. Box 80203, Jeddah 21589, Saudi Arabia, Department of Mathematics, Faculty of Science, Mansoura University, Mansoura 35516, Egypt

E-mail address: emelsayed@mans.edu.eg, emmelsayed@yahoo.com 